\title{
Micro Insert: A Prototype Full-Ring PET Device for Improving the Image Resolution of a Small- Animal PET Scanner
}

\author{
Heyu Wu ${ }^{1,2}$, Debashish $\mathrm{Pal}^{3}$, Tae Yong Song ${ }^{1}$, Joseph A. O’Sullivan ${ }^{4}$, and Yuan-Chuan Tai ${ }^{1,5}$ \\ ${ }^{I}$ Mallinckrodt Institute of Radiology, Washington University in St. Louis, St. Louis, Missouri; ${ }^{2}$ Institute of Modern Physics, Chinese \\ Academy of Science, Lanzhou, China; ${ }^{3}$ Department of Biomedical Engineering, Washington University in St. Louis, St. Louis, Missouri; \\ ${ }^{4}$ Department of Electrical and System Engineering, Washington University in St. Louis, St. Louis, Missouri; and ${ }^{5}$ Alvin J. Siteman Cancer \\ Center, Washington University School of Medicine, St. Louis, Missouri
}

\begin{abstract}
A full-ring PET insert device should be able to enhance the image resolution of existing small-animal PET scanners. Methods: The device consists of 18 high-resolution PET detectors in a cylindric enclosure. Each detector contains a cerium-doped lutetium oxyorthosilicate array $(12 \times 12$ crystals, $0.72 \times 1.51 \times 3.75 \mathrm{~mm}$ each) coupled to a position-sensitive photomultiplier tube via an optical fiber bundle made of $8 \times 16$ square multiclad fibers. Signals from the insert detectors are connected to the scanner through the electronics of the disabled first ring of detectors, which permits coincidence detection between the 2 systems. Energy resolution of a detector was measured using a ${ }^{68} \mathrm{Ge}$ point source, and a calibrated ${ }^{68} \mathrm{Ge}$ point source stepped across the axial field of view (FOV) provided the sensitivity profile of the system. $A^{22} \mathrm{Na}$ point source imaged at different offsets from the center characterized the in-plane resolution of the insert system. Imaging was then performed with a Derenzo phantom filled with $19.5 \mathrm{MBq}$ of ${ }^{18} \mathrm{~F}$-fluoride and imaged for $2 \mathrm{~h}$; a 24.3-g mouse injected with $129.5 \mathrm{MBq}$ of ${ }^{18} \mathrm{~F}$-fluoride and imaged in 5 bed positions at 3.5 $\mathrm{h}$ after injection; and a 22.8-g mouse injected with 14.3 MBq of ${ }^{18} \mathrm{~F}-\mathrm{FDG}$ and imaged for $2 \mathrm{~h}$ with electrocardiogram gating. Results: The energy resolution of a typical detector module at 511 $\mathrm{keV}$ is $19.0 \% \pm 3.1 \%$. The peak sensitivity of the system is approximately $2.67 \%$. The image resolution of the system ranges from 1.0- to 1.8-mm full width at half maximum near the center of the FOV, depending on the type of coincidence events used for image reconstruction. Derenzo phantom and mouse bone images showed significant improvement in transaxial image resolution using the insert device. Mouse heart images demonstrated the gated imaging capability of the device. Conclusion: We have built a prototype full-ring insert device for a small-animal PET scanner to provide higher-resolution PET images within a reduced imaging FOV. Development of additional correction techniques are needed to achieve quantitative imaging with such an insert.
\end{abstract}

Received Dec. 19, 2007; revision accepted Jun. 20, 2008.

For correspondence or reprints contact: Yuan-Chuan Tai, Washington University School of Medicine, Campus Box 8225, 510 S. Kingshighway Blvd., St. Louis, MO 63110.

E-mail: taiy@wustl.edu

COPYRIGHT @ 2008 by the Society of Nuclear Medicine, Inc.
Key Words: small-animal PET; high-resolution PET; PET insert; animal imaging

J Nucl Med 2008; 49:1668-1676

DOI: 10.2967/jnumed.107.050070

$\mathbf{H}$ igh-resolution PET scanners dedicated to small-animal imaging have been developed by several research groups since the 1990s (1-11). Combining high-resolution and quantitative imaging capability, small-animal PET has been a driving force behind the development of molecular imaging that brings together scientists from different disciplines to study biologic effects at the molecular level $(12,13)$. Smallanimal PET has also been adopted by the pharmaceutical industry to study pharmacokinetics and pharmacodynamics to accelerate development of new drugs (14). The increasing demand for small-animal PET has led to commercialization of several small-animal PET technologies (15). Current technologic research and development is focused on further improvement of the resolution or sensitivity of small-animal PET systems (16).

Most commercial small-animal PET scanners use inorganic scintillators for $\gamma$-ray detection, a proven technology that provides good image resolution and system sensitivity at a reasonable cost. To achieve high spatial resolution, the scintillation crystals are usually cut into small cross-sections of 1-2 mm. To maintain good detection efficiency, the crystal length is typically around $10 \mathrm{~mm}$ or longer. To offer high system sensitivity, the radius of the detector ring is usually small to maximize the solid angle coverage of the detectors. A common dilemma is that as a design improves one aspect of the scanner performance (such as sensitivity), it often degrades other aspects of system performance (such as image resolution). It is, therefore, difficult to improve multiple aspects of system performance without a dramatic change in detector technology or system design (17), which would inevitably increase the complexity and cost of a PET system. As a result, most commercial small-animal PET scanners 
have an image resolution between 1.3- and 2-mm full width at half maximum (FWHM) and a system sensitivity of $2 \%-$ $10 \%$ near the center of the field of view (FOV). Although these systems are adequate for many imaging applications, submillimeter-resolution PET systems are highly desirable to better image transgenic mice that are widely used to study human diseases. The fundamental limit of PET image resolution with short-range positron sources such as ${ }^{18} \mathrm{~F}$ was estimated to be around 0.6-mm FWHM (assuming a 10-cm system diameter) (18), suggesting room for improvement in the small-animal PET system design.

We have proposed a novel geometry for PET, the "virtualpinhole PET geometry" (19), that uses high- and lowresolution $\gamma$-ray detectors in a coincidence detection system to provide high-resolution PET images. We recently demonstrated the feasibility of using a high-resolution PET detector, rotated inside an existing small-animal PET scanner, to implement the virtual-pinhole PET geometry and obtain higher image resolution from a microPET F-220 system (Siemens Molecular Imaging, Inc.) (20). The current study describes the design and initial results of a full-ring micro insert device that can be integrated into a microPET F-220 scanner to improve its image resolution within a reduced FOV.

\section{MATERIALS AND METHODS}

\section{Commercial microPET F-220 Scanner}

The microPET F-220 scanner used in this study normally consists of 168 detector modules arranged in 4 rings ( $258 \mathrm{~mm}$ in diameter). Each detector module is composed of a lutetium oxyorthosilicate (LSO) array of $12 \times 12$ crystals, each measuring $1.51 \times 1.51 \times 10.00$ $\mathrm{mm}$; an optical fiber bundle with $8 \times 8$ square fibers; and a positionsensitive photomultiplier tube (PS-PMT). The pitch of the crystals is $1.59 \mathrm{~mm}$ in both transverse and axial directions. The output signals of each detector module are processed by an independent channel of electronics in the base cabinet. A coincidence processor compares singles events from all detectors and transfers qualified coincidence events to the host computer to be stored in a list-mode file. List-mode data are then sorted into sinograms and subsequently reconstructed into tomographic images (21). Two gating signals can be inserted into the data stream to support imaging studies with cardiac or respiratory gating.

\section{Micro Insert System}

The micro insert system contains 18 high-resolution detector modules, circularly mounted to a custom aluminum base to form a detector ring with a 56-mm inner diameter (Fig. 1A). A cover made of aluminum and a thin stainless steel sheet shields the detectors from ambient light. The base of the system can be mounted to the back of the microPET F-220 scanner after the rotation mechanism of the transmission source is removed from the scanner (Fig. 1B). The animal port of the insert device is $5.4 \mathrm{~cm}$ in diameter. A rodent-only animal bed is used when the insert device is attached to the scanner (Fig. 1C). The insert detector ring is concentric to and axially aligned with the third detector ring in the scanner, as shown in Figure 1D. Two alignment pins in the back of the scanner permit the insert to be attached to the scanner repeatedly, with high precision. The alignment along the axial direction was adjusted by thin washers after a series of calibration measurements using a ${ }^{22} \mathrm{Na}$ point source to identify the necessary axial offset between the 2 systems.

Each detector module in the insert consists of an LSO array, a coherent optical fiber bundle, and a PS-PMT. The LSO array is made of $12 \times 12$ crystals, each measuring $0.72 \times 1.51 \times 3.75 \mathrm{~mm}$. With reflective film $(3 \mathrm{M})$ inserted between the crystal elements, the crystal pitch is 0.80 and $1.59 \mathrm{~mm}$ in the transverse and axial directions, respectively. Compared with the pitch of the microPET F-220 scanner, the axial crystal pitch of the insert detector remains unchanged, and the transaxial crystal pitch of the insert detector is reduced by $50 \%$ to improve the in-plane image resolution. The fiber bundles were made in-house and have a $90^{\circ}$ bend to allow the PS-PMT to be positioned outside the imaging FOV of the micro insert device. This design reduces the attenuation of $\gamma$-rays by the light detectors (PS-PMT). Each fiber bundle is made of $8 \times 16$ multicladding square
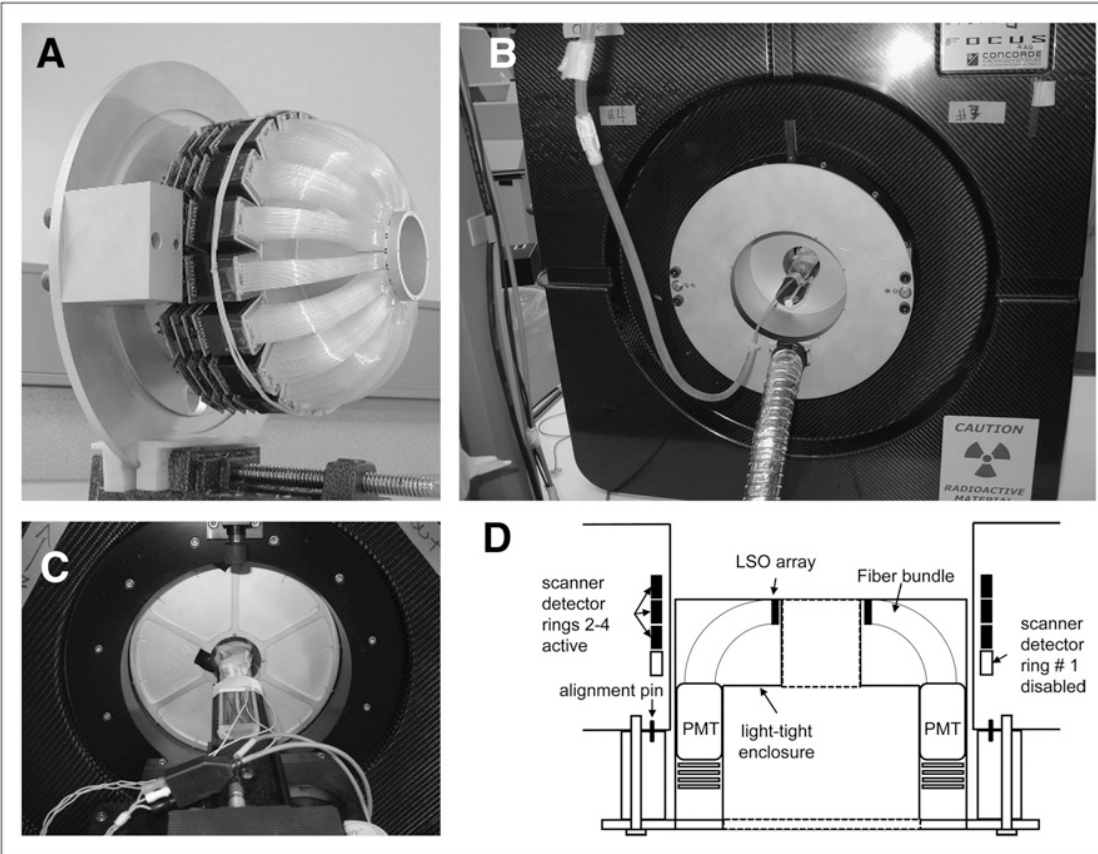

FIGURE 1. Photographs of micro insert device. (A) Device with its cover removed to show 18 detector modules arranged in ring. Each detector module consists of LSO crystal array, plastic optical fiber bundle that bends $90^{\circ}$, and PS-PMT and its associated readout boards. (B) Device mounted to back of microPET F-220 scanner after removal of transmission source holder. All signal cables and high-voltage cable are connected to base cabinet through light-tight hose. (C) Front view of scanner with micro insert attached. Animal port opening is reduced to $5.4 \mathrm{~cm}$ in diameter. (D) Schematic illustrating position of insert detector ring relative to detector rings in scanner. 
plastic fibers (BCF-98MC; Saint-Gobain) of $1.2 \times 1.2 \mathrm{~mm}$ crosssections, with a length ranging from 45 to $60 \mathrm{~mm}$ for the innermost to the outermost layer of fibers, respectively. In contrast, the fiber bundles in the microPET F-220 scanner are made of 100-mm long square fibers of $2.4 \times 2.4 \mathrm{~mm}$ cross-sections. The front end of a fiber bundle has a cross-section of $9.6 \times 19.2 \mathrm{~mm}$ that matches the dimension of the LSO crystal array. Fibers spread out at the back end of a bundle to match the active area of a PMT $\left(\sim 20 \times 20 \mathrm{~mm}^{2}\right)$. Scintillation light from a $3 \times 3$ subarray of LSO crystals is coupled to the PS-PMT by a group of $2 \times 4$ optical fibers with light sharing between adjacent fibers (Fig. 2A). This light-sharing scheme allows us to use larger optical fibers, which are easier to handle, and still be able to identify all crystals in the flood image of a detector. Figure 2B shows the flood image of a typical detector module and a profile through a column of crystals. The PS-PMT and its associated electronics, such as charge-division resistor readout, are identical to those used in the microPET F-220 scanner, permitting us to directly feed the outputs of the micro insert detectors into the microPET F-220 scanner.

All PS-PMTs of the 18 detectors are powered by an external highvoltage power supply ( 556 ; Ortec). The $\pm 5 \mathrm{~V}$ for powering the readout boards are drawn from the scanner through the same ribbon cables that transmit the output signals. To establish coincidence detection between the scanner and our insert device, the entire first ring (of a total of 4 rings) of detectors in the scanner is disabled to make 42 channels of readout electronics available for receiving signals from the insert device. Because this prototype system has only 18 detectors, 18 of the 42 channels are currently used for the insert detectors. The remaining 24 channels of electronics offer the potential to increase the number of detectors in the insert device to increase its axial FOV in the future.

\section{Coincidence Detection and Image Reconstruction}

Originating within the imaging FOV are $511-\mathrm{keV} \gamma$-rays that may interact with detectors in the insert or penetrate the insert device (because the crystals in the insert detectors are only $3.75 \mathrm{~mm}$ thick) and become detected by the detectors in the scanner. Because the microPET F-220 scanner acquires data in 3 dimensions, coincidences can be registered between detectors in any combination of the 4 rings. Therefore, coincidences can be detected between insert detectors that are read out by electronics for ring 1 (insert-insert, or II, events), between original scanner detectors that are read out by electronics for rings 2-4 (scanner-scanner, or SS, events), and between insert detectors in ring 1 and scanner detectors in rings 2-4 (insert-scanner, or IS, events).

We previously demonstrated through Monte Carlo simulation that these 3 types of coincidences lead to different image resolutions (22). We also developed analytic and statistical image reconstruction algorithms for PET insert devices by modeling the unique virtual-pinhole PET geometry (23). The 2-dimensional filtered backprojection (FBP) algorithm was used to reconstruct point source images in the image resolution measurements below. A 3-dimensional ordered-subset expectation maximization (OSEM) reconstruction algorithm was developed and used for all phantom and small-animal imaging experiments (Debashish Pal, Joseph A. O'Sullivan, Heyu Wu, and Yuan-Chuan Tai, unpublished data, 2008). The system matrix can be described as a normalized point spread function computed by numeric integration over the front surfaces of a detector pair, taking into account the difference in detector dimensions and ring radii of the insert and the scanner. All effects on the absolute detector efficiency are included in the normalization. There are a total of 3 system submatrices, each with a unique spatially varying detector resolution model for the corresponding type of coincidence (II, IS, and SS). This algorithm allows us to reconstruct images from individual types of coincidence events and use all 3 types of events to jointly estimate an image volume such that the image resolution can be improved while the overall sensitivity of the system can be maximized. All coincidence data were first corrected for randoms (estimated from the product of singles counting rate of individual detectors) and subsequently

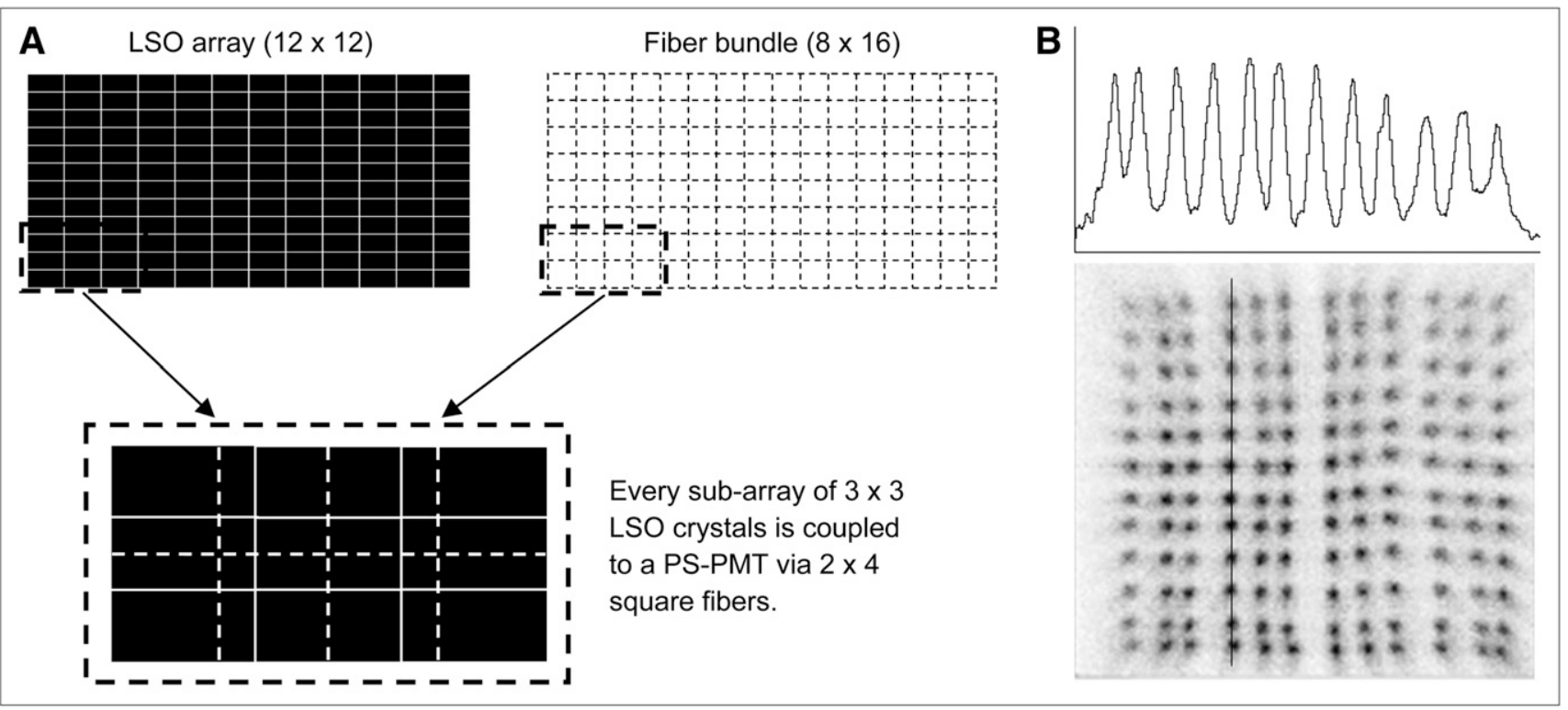

FIGURE 2. (A) Drawings illustrating light-sharing pattern used to couple scintillation light from LSO crystals to PS-PMT. Crosssection of rectangular LSO array $(12 \times 12$ crystals) matches dimension of fiber bundle $(8 \times 16$ square fibers). Each $3 \times 3$ subarray of LSO crystals is coupled to PMT via $2 \times 4$ subarray of square fibers. (B) Flood image of typical micro insert detector module and profile through fourth column of crystals in image. 
normalized for variation in detection efficiency on the basis of a component-based normalization technique (24) using a sinogram of a ring source phantom acquired by the micro insert system.

\section{Evaluation of Basic Performance}

Energy resolution of the insert detector module was measured by standard nuclear instrument modules and a 12-bit analog-todigital converter in a workstation. A flood image similar to that in Figure $2 \mathrm{~B}$ was acquired using a ${ }^{68} \mathrm{Ge}$ point source in singles mode. Crystals were identified to create a lookup table. Energy spectra of individual crystals were measured to compute the energy resolution at $511 \mathrm{keV}$ for each crystal in a typical detector module.

All performance experiments with insert detectors read out by scanner electronics were performed using a 250 - to $750-\mathrm{keV}$ energy window and a 6-ns coincidence timing window. All images were reconstructed to form $256 \times 256$ matrices with a $0.2 \times 0.2 \times$ $0.8 \mathrm{~mm}$ voxel dimension.

Sensitivity of the system was measured with a calibrated ${ }^{68} \mathrm{Ge}$ point source of $2.28 \mathrm{MBq}$ stepping across the axial FOV along the central axis of the scanner. At each location, coincidence events were collected for $120 \mathrm{~s}$ and sorted into 3 sinograms on the basis of the type of coincidences (II, SS, and IS). The total number of coincidences in each type of sinogram was normalized to the activity of the source to compute the absolute sensitivity for each type of coincidence. The sensitivity profiles were plotted for all 3 types of events as a function of axial location.

Tangential and radial resolutions of the micro insert device were measured using a $1.6-\mathrm{MBq}^{22} \mathrm{Na}$ point source (nominal diameter of $0.5 \mathrm{~mm}$, embedded in a 25.4-mm-diameter, 8-mm-thick Lucite disk). The axial resolution was not evaluated because the crystal pitch of the micro insert device is identical to the original microPET F-220 system, and we did not expect the axial resolution of the system to be significantly different from the original scanner near the central axis of the scanner (although the axial resolution of this system may potentially degrade faster than that of the original scanner when a source is away from the central axis of the FOV). The source was first positioned at the center of the FOV to acquire images and then was repositioned at different radial offsets for subsequent imaging experiments. The 3 types of coincidence events (II, SS, and IS) were sorted into 3 sinograms using single-slice rebinning (25) and subsequently reconstructed individually using a 2-dimensional FBP algorithm with a ramp filter cutoff at the Nyquist frequency. Tangential and radial profiles through the peak in each point source image were extracted to measure the tangential and radial resolutions (expressed in FWHM and full width at tenth maximum) of individual types of events. Images of all point sources were also reconstructed by a 2-dimensional OSEM algorithm that jointly estimates the image using all 3 types of coincidences. This combined image demonstrates the potential improvement in image resolution for point source-like objects. The resolution of images reconstructed using different algorithms (FBP vs. OSEM in this case) should not be directly compared.

\section{Phantom Study}

A custom-made, micro-Derenzo hot-rod phantom was scanned to evaluate the resolution enhancement produced by the micro insert system. The phantom has an inner diameter of $32 \mathrm{~mm}$ and contains fillable hot rods of different sizes $(0.80,1.00,1.25,1.50,2.00$, and $2.50 \mathrm{~mm}$ ) arranged into 6 segments. The spacing between adjacent rods in each segment is twice the rod diameter. The phantom was filled with $19.5 \mathrm{MBq}(0.53 \mathrm{mCi})$ of ${ }^{18} \mathrm{~F}-\mathrm{FDG}$ and scanned for 120 min. List-mode data were sorted into 3 fully 3-dimensional sinograms on the basis of the type of coincidence events. Images were reconstructed with the 3-dimensional OSEM algorithm for each type of coincidence. A single-image volume was also jointly estimated by using either the II plus IS type of coincidence events or all 3 types of events.

\section{Small-Animal Studies}

A $24.3-\mathrm{g}$ mouse was injected with $129.5 \mathrm{MBq}(3.5 \mathrm{mCi})$ of ${ }^{18} \mathrm{~F}-$ fluoride and imaged in the micro insert device in 5 bed positions starting at $3.5 \mathrm{~h}$ after injection. The acquisition time was $30 \mathrm{~min}$ for the initial bed position and adjusted for the radioactive decay of ${ }^{18} \mathrm{~F}$ for other bed positions to obtain the equivalent number of decays per bed position. List-mode data were sorted into 3 fully 3-dimensional sinograms and reconstructed as described above for the phantom imaging study to obtain 5 types of images using either individual coincidence types or combined datasets. Image slices within the central $2 \mathrm{~cm}$ of the axial FOV were extracted from individual bed positions and stitched together to form the whole-body mouse bone images.

A 22.8-g mouse was positioned inside the micro insert device with the heart of the mouse centered in the imaging FOV. The mouse was imaged for 120 min immediately after the injection of $14.3 \mathrm{MBq}$ $(386 \mu \mathrm{Ci})$ of ${ }^{18} \mathrm{~F}-\mathrm{FDG}$. An electrocardiogram signal was fed into the scanner and inserted into the data stream. List-mode data were sorted into 3 types of sinograms on the basis of the type of coincidence events, with 8 gates each (a total of 24 fully 3-dimensional sinograms). Images at different gates of the cardiac cycle were reconstructed with a 3-dimensional OSEM algorithm using either the SS type of coincidence (corresponding to original scanner resolution) or the IS plus II types of coincidence combined (corresponding to high resolution).

\section{RESULTS}

\section{Basic Performance of Micro Insert}

Energy resolution of 144 crystals in a typical detector module at $511 \mathrm{keV}$ ranges from $13.9 \%$ to $31.6 \%$, with a mean $\pm \mathrm{SD}$ of $19.0 \% \pm 3.1 \%$. Figure $3 \mathrm{~A}$ shows a typical detector module and the worst energy spectra of a typical detector module.

The sensitivity of the microPET F-220 system with micro insert attached is shown in Figure 3B for each of the 3 types of coincidence and for all 3 types together (providing the overall system sensitivity). The previously reported sensitivity profile of the microPET F-220 system is also included for comparison. A correction technique for scatter remains to be developed; therefore, the results were only corrected for random coincidences but not for scatter coincidences. The sensitivity profile of the II events is similar to that of a singlering PET scanner with an axial FOV of $19.2 \mathrm{~mm}$. The profile has a triangular shape with a peak sensitivity of approximately $0.4 \%$ at the center. The sensitivity profile of the IS events has a peak of approximately $1.2 \%$, with a slightly extended axial FOV because the coincidence is between a 1-ring insert and a 3-ring microPET F-220 scanner. The sensitivity profile of SS events is different from that of a typical PET scanner. Discontinuity at approximately -22 $\mathrm{mm}$ from the center of the axial FOV due to the attenuation of $\gamma$-rays by the aluminum front cover of the insert device is 

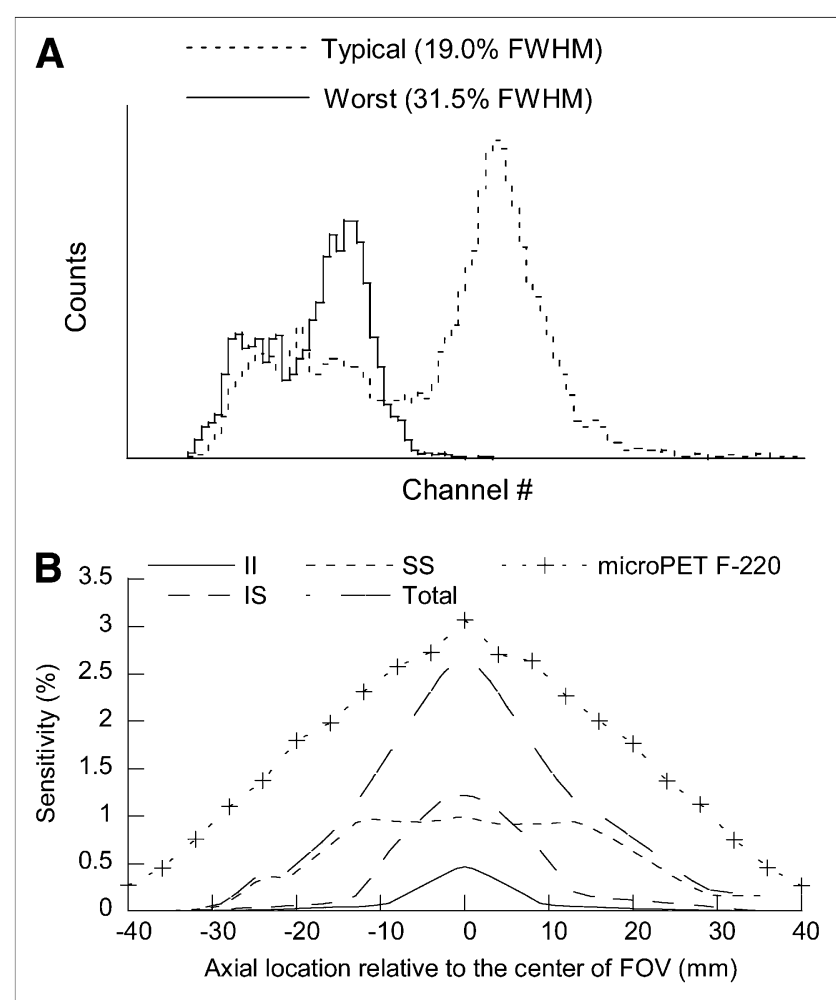

FIGURE 3. (A) Energy spectra of typical and worst crystal in typical insert detector module. (B) Sensitivity of microPET F-220 scanner with micro insert device attached, measured with 250$750 \mathrm{keV}$ energy window and 6-ns timing window. Sensitivity profile of original microPET F-220 scanner is included for comparison.

observed. The middle section of the profile is lower than that of a typical 3-ring PET scanner because $\gamma$-rays from the source may interact with detectors in the micro insert and become absorbed before they can interact with the scanner. The rear section of the sensitivity profile does not reach zero because of scattered events caused by the optical fiber bundles and the enclosure of the insert device. The summed sensitivity profile of the system shows that the peak sensitivity of the system is approximately $2.67 \%$ (before scatter correction). This peak sensitivity is approximately $11 \%$ lower than the sensitivity of the original microPET F-220 scanner (3.0\% at the center of the FOV) but slightly higher than that of a small-animal PET scanner with 3 active rings of the microPET Focus detector (the condition under which the microPET F-220 scanner operates during our experiments), assuming that the peak sensitivity of a 3-ring system is approximately $75 \%$ of a 4-ring system.

Figure 4A shows the FBP-reconstructed point source images of the SS (top row), IS (second row), and II events (third row) and an OSEM-reconstructed image using all 3 types of events (last row). The improvement in image resolution can be seen clearly from the IS and II images, as well as the jointly estimated image using all events. Figure 4B shows the tangential and radial resolutions of the system as a function of radial offset, directly measured from images in Figure 4A without compensating for the dimension of the ${ }^{22} \mathrm{Na}$ point source. The resolution of the SS image is similar to that of the microPET F-220 scanner that we previously reported. The resolution of IS and II images ranges from 1.3- to 1.5-mm FWHM and from 1.0- to 1.2-mm FWHM, respectively, for the central 2-cm FOV. The jointly estimated image using OSEM algorithm clearly achieves submillimeter resolution for highly localized sources.

Table 1 summarizes the basic performance of the micro insert system and the microPET F-220 scanner.

\section{Phantom Study}

Figure 5 shows the miniature Derenzo phantom image reconstructed in 5 ways. Among the images reconstructed using individual types of coincidence event, the SS image has the lowest resolution and high statistics. The II image has the highest resolution and the lowest statistics. The IS image has intermediate resolution and high statistics. The image that combines II and IS types of events has a resolution close to that of the II image and significantly better noise characteristics than II events alone. The image that combines all 3 types of events appears to have slightly better resolution than the IS image and the best noise properties of all the images. All images (except SS) have higher resolution than that of the original scanner, which is close to the resolution of the SS image.

\section{Small-Animal Studies}

The transverse bone images reconstructed using individual types of coincidence events show once again that the SS image has the lowest resolution and the II image has the highest resolution (Fig. 6). Comparison of individually reconstructed images and jointly estimated images results in conclusions similar to the ones found for the phantom. The maximum-intensity projection image of individually versus jointly reconstructed images shows less-significant improvement in image resolution because the axial resolution of the micro insert system is the same as the original microPET F-220 scanner. Therefore, only the resolution in the horizontal direction (corresponding to transverse image resolution) is improved by the use of the micro insert. Discontinuity in activity distribution can be seen in the II image along the axial direction because the sensitivity profile of II data approaches zero near the edge of the highresolution imaging FOV. This discontinuity becomes less visible when the image is reconstructed using other types of coincidence data or using more than one type of coincidence event.

Figure 7 shows the ${ }^{18} \mathrm{~F}-\mathrm{FDG}$ images of a mouse heart at end-systole and end-diastole. In the top row, images were reconstructed using the SS type of coincidence only. In the bottom row, images were reconstructed using both II and IS types of coincidence. The improvement in image resolution is clear. Myocardium walls of both left and right ventricles are better delineated and defined in the high-resolution images using both II and IS events. 

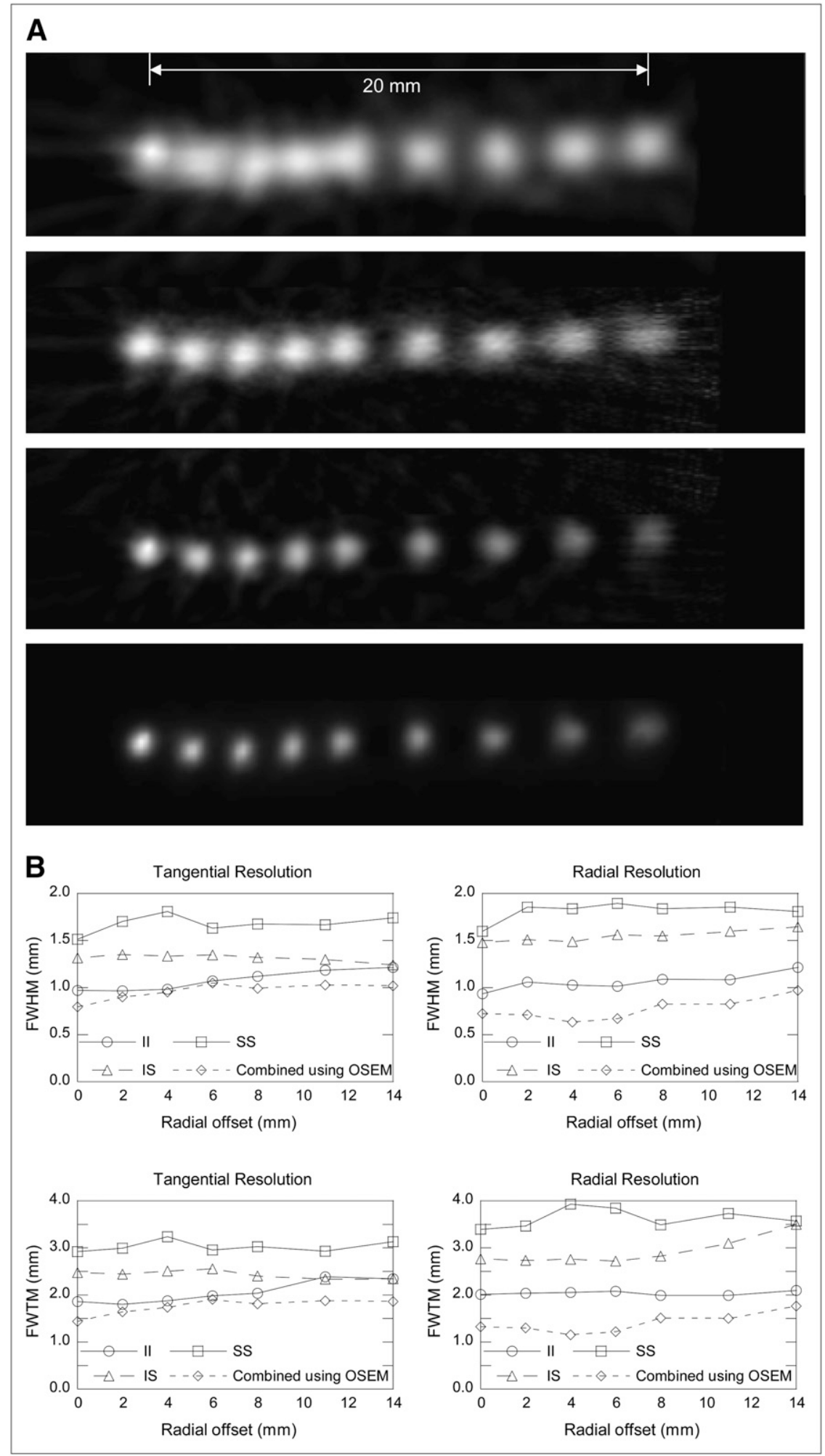

FIGURE 4. (A) Images of point sources at different radial offsets from center of FOV (located near left-most point). First 3 rows of images are reconstructed by FBP using SS (top row), IS (second row), and II events (third row). Image in last row is reconstructed by OSEM using all 3 types of event. (B) Tangential and radial resolutions of system are directly measured from images and plotted as function of radial offset without compensating for dimension of ${ }^{22} \mathrm{Na}$ point source. FWTM = full width at tenth maximum.

\section{DISCUSSION}

The energy resolution of detectors in the micro insert is slightly worse than that of typical detectors in a microPET F-220 scanner. The degradation in energy resolution appears to be more significant for crystals near the edges of an array.
This significant degradation may be the result of increased optical cross-talk in the in-house-built fiber bundles, when compared with those in the microPET F-220 scanner that use reflective films between adjacent fibers, and microscopic damage to the cladding of the multiclad fiber due to thermal 
TABLE 1

Comparison of Micro Insert System and microPET F-220 Scanner

\begin{tabular}{|c|c|c|}
\hline Characteristic & $\begin{array}{l}\text { Micro insert } \\
\text { system }\end{array}$ & $\begin{array}{c}\text { microPET } \\
\text { F-220 }\end{array}$ \\
\hline \multicolumn{3}{|l|}{ Image resolution (FWHM) } \\
\hline SS events & $1.7-1.8 \mathrm{~mm}$ & $1.7-1.8 \mathrm{~mm}$ \\
\hline IS events & $1.3-1.5 \mathrm{~mm}$ & NA \\
\hline Il events & $1.0-1.2 \mathrm{~mm}$ & NA \\
\hline $\begin{array}{l}\text { Sensitivity at the center of } \\
\text { FOV (250- to } 750-\mathrm{keV} \\
\text { energy window and 6-ns } \\
\text { timing window) }\end{array}$ & $2.67 \%$ & $3.0 \%$ \\
\hline Energy resolution & $19.0 \%$ & $18.5 \%$ \\
\hline \multicolumn{3}{|l|}{ FOV } \\
\hline Transaxial & $\sim 4 \mathrm{~cm}$ & $20 \mathrm{~cm}$ \\
\hline \multirow[t]{2}{*}{ Axial } & $5.7 \mathrm{~cm}$ total with & $7.6 \mathrm{~cm}$ \\
\hline & $\begin{array}{l}\text { central } 2 \mathrm{~cm} \text { with } \\
\text { higher resolution }\end{array}$ & \\
\hline
\end{tabular}

$\mathrm{NA}=$ not applicable

shock caused by the heat treatment during the bending process. Such damage, if it exists, could cause light leakage that could further increase optical cross-talk and loss of signal. We do not anticipate the light leakage to be significantly different between the inner and outer layers of fibers because the smallest radius of the bent fibers $(47 \mathrm{~mm})$ is approximately 40 times the cross-section of the fiber, which is significantly larger than what is recommended ( 5 times the fiber radius) by the manufacturer to avoid significant damage to the cladding. This hypothesis is supported by the fact that the energy resolution does not closely correlate with the radial location of the crystals in an array, which are coupled to PMT by fibers of different curvatures.

Although the bent fiber bundle design allows us to position the PS-PMT outside the FOV of the system to reduce attenuation of $\gamma$-rays by light detectors, the plastic fiber bundles and the metal enclosure still contribute to attenuation and scatter of $\gamma$-rays. It may be advantageous to use semiconductor photon detectors (such as an avalanche photodiode or a silicon photomultiplier) for future PET insert detectors to minimize the physical size of the device and to avoid the need for optical fiber bundles. Alternatively, semiconductor-based detectors such as cadmium-zinc-telluride or mercury-iodide could be used instead of scintillator-based detectors to achieve higher spatial and energy resolution. One consideration, however, is that the matching of the amplitude and shape of detector signals to existing electronics in the microPET F-220 scanner could be a challenge if different types of detectors are used. However, if a PET scanner is designed to accept a specific type of detector signal from an insert device, then in theory there should be no restriction on the type of detector that could be used in the insert.

When the micro insert is attached to the system, the axial FOV is reduced from the original from 7.6 to $5.7 \mathrm{~cm}$, with the high-resolution imaging capability within the central 2-cm axial FOV only. For the mouse bone scan using ${ }^{18} \mathrm{~F}$-fluoride, high-resolution images of the entire body were obtained by stitching together images within the central $2 \mathrm{~cm}$ of the axial FOV from 5 bed positions. For most studies that require dynamic or gated imaging capability, one may choose to position the organ of interest in the center of the axial FOV to obtain higher-resolution images for the organ yet still obtain regular resolution images for most of the rest of the body. For example, in the ${ }^{18} \mathrm{~F}-\mathrm{FDG}$ cardiac gated study, the mouse was imaged in a single bed position with the heart centered in the FOV. For studies that need higherresolution images of an organ of interest instead of a large FOV that covers the entire mouse body, the micro insert device could be used to locally enhance image resolution. In other applications that require dynamic imaging of the entire mouse body, the micro insert device may not be beneficial at all. Therefore, this device should only be considered as an accessory to enhance the imaging capability and functionality of the original scanner and is not meant to replace the standard configuration of the original scanner.

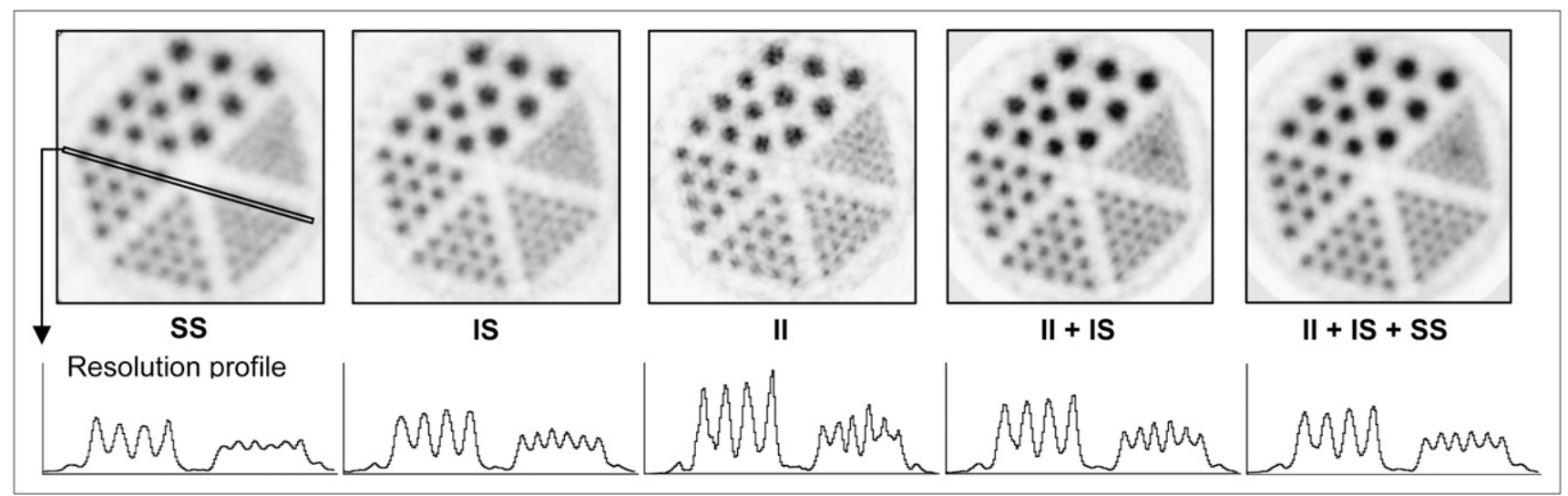

FIGURE 5. micro-Derenzo phantom imaged by micro insert and reconstructed by OSEM algorithm using different types of coincidence events. Diameters of fillable rods in phantom are $0.8,1.0,1.25,1.5,2.0$, and 2.5 mm, respectively. Rods in given section are separated by twice their diameter. Profiles through 1- and 1.5-mm-diameter rods clearly show improvement in image resolution when coincidence events measured by insert are included for image reconstruction. 
A

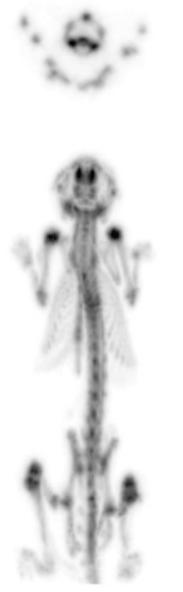

B
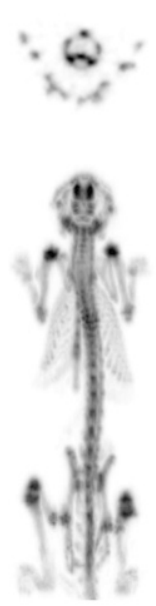

C
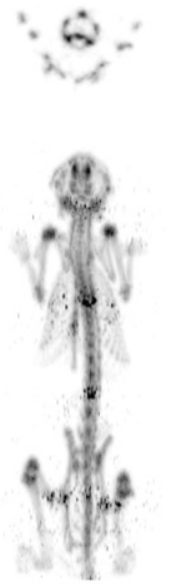

D
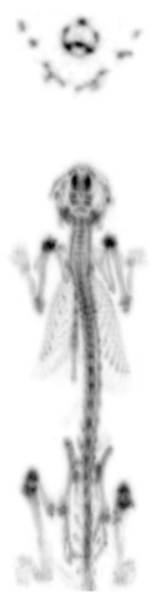

E
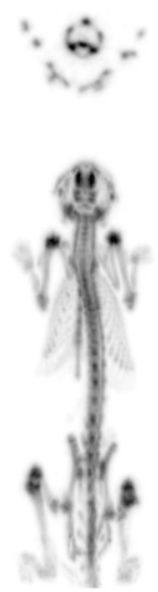

FIGURE 6. A 24.3-g mouse imaged by micro insert device in 5 bed positions starting at $3.5 \mathrm{~h}$ after $129.5-\mathrm{MBq}$ ${ }^{18} \mathrm{~F}$-fluoride injection. Images are reconstructed by OSEM algorithm using different types of coincidence events: SS (A), IS (B), II (C), II + IS (D), and II + IS + SS (E). Transverse images are shown in top row, and maximum-intensity projection images are shown in bottom row.
As can be seen in Figures 5 and 6, images can be reconstructed by combining different types of coincidence events. If a study requires the highest sensitivity from a system with moderate resolution enhancement, all 3 types of events should be combined to reconstruct the image. If a study has sufficient counting statistics and requires the highest achievable image resolution, II events might be used alone. Alternatively, II and IS events could be combined to achieve intermediate sensitivity and significant resolution improvement. With the micro insert system, the data acquisition and image reconstruction protocol can be adjusted to match the counting statistic and performance requirements of a specific study after the system is thoroughly characterized.

The current implementation of the micro insert device requires the transmission source holder to be removed from the system before use. Although the transmission scan can be obtained before setting up the micro insert device for the emission scan, it is not practical to switch between the 2 modes of operation because it takes approximately $20 \mathrm{~min}$ to set up the micro insert device and another $20 \mathrm{~min}$ to restore the scanner to its original function. A potential implementation of a micro insert device to retain the transmission

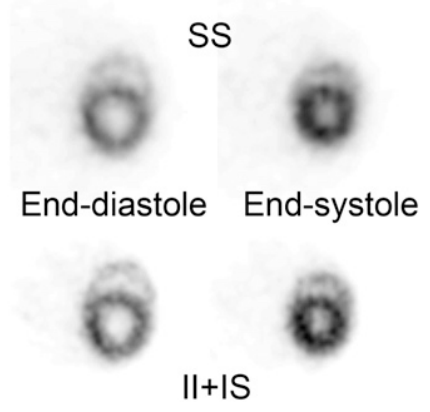

FIGURE 7. A 22.8-g mouse imaged by micro insert device with electrocardiogram gating after $14.3-\mathrm{MBq}{ }^{18} \mathrm{~F}-\mathrm{FDG}$ injection. Images in top row are reconstructed using SS type of event, which has image resolution similar to that of original scanner. Images in bottom row are reconstructed using II and IS events, which show improved image resolution that better delineates left and right ventricles of mouse heart. imaging capability of a small-animal PET scanner is to design a device that can be attached to the small-animal bed. This approach is, however, likely to require 3-dimensional movement control for the small-animal bed holder, which is beyond the capability of the current microPET F-220 scanner. Alternatively, a flexible image reconstruction algorithm that can compensate for misalignment of the 2 systems would permit the micro insert device to be mounted on the current small-animal bed holder without requiring the 2 systems to be concentric. Such flexibility would have a cost, however. When the 2 systems are not concentric, the system is no longer circularly symmetric. This significantly increases the size of the system matrix, and such an increase requires more computation for system matrix generation, larger disk storage space, and longer image reconstruction time due to constant memory swapping to disk. A more practical approach may be through the use of coregistered CT images to perform calculated attenuation correction, which is used on a routine basis at our institution if a transmission scan is not practical for certain imaging protocols.

Although we have implemented the component-based normalization technique for this micro insert system, many correction techniques still must be developed to produce fully quantitative PET images using this system. For example, the CT-based attenuation correction needs to be implemented and tested. The scatter correction technique needs to be developed to correct scatter contribution from the micro insert device and from the object being imaged. The dead time of the insert system may be significant because its detectors are more than 4 times closer to the mouse than are standard detectors in the scanner. Therefore, the dead-time characteristics of the insert device need to be carefully analyzed. A complete characterization of the system performance and quantitative accuracy will require that these correction techniques are developed.

\section{CONCLUSION}

We have developed a prototype full-ring micro insert device for the microPET F-220 system. The integrated 
system has a peak system sensitivity of $2.67 \%$ and an imaging FOV of $5.7 \mathrm{~cm}$ in the axial direction and $4 \mathrm{~cm}$ in the transaxial direction. The transaxial image resolution can be improved to approximately 1-mm FWHM within the central 2-cm axial FOV. A 3-dimenstional OSEM image reconstruction algorithm has been developed to combine II, IS, and SS coincidence events to jointly estimate an image volume and achieve high-resolution and high-sensitivity mouse imaging simultaneously. The system is capable of dynamic and gated imaging. Preliminary phantom and mouse imaging studies show significant improvement in image resolution in the transverse direction. This type of PET insert device may increase the versatility of existing PET scanners, but additional correction techniques must be developed.

\section{ACKNOWLEDGMENTS}

We thank Drs. Stefan B. Siegel and Danny F. Newport of Siemens Molecular Imaging, Inc., for their inspiring discussion; Dr. Sergey Komarov, Dennis Tapella, and John Kreitler; and the staff of the microPET Lab of Washington University in St. Louis for their technical support. This work was supported in part by the National Cancer Institute of the National Institutes of Health (grants R24-CA83060, R33CA-110011, and P30-CA91842) and by the Susan G. Komen for the Cure (grant BCTR0601279).

\section{REFERENCES}

1. Bloomfield PM, Rajeswaran S, Spinks TJ, et al. The design and physical characteristics of a small animal positron emission tomograph. Phys Med Biol. 1995;40:1105-1126

2. Lecomte R, Cadorette J, Rodrigue S, et al. Initial results from the Sherbrooke avalanche photodiode positron tomograph. IEEE Trans Nucl Sci. 1996;43: 1952-1957.

3. Cherry SR, Shao Y, Silverman RW, et al. MicroPET: a high resolution PET scanner for imaging small animals. IEEE Trans Nucl Sci. 1997;44:1161-1166.

4. Watanabe M, Okada $\mathrm{H}$, Shimizu $\mathrm{K}$, et al. A high resolution animal PET scanner using compact PS-PMT detectors. IEEE Trans Nucl Sci. 1997;44:1277-1282.

5. Del Guerra A, Di Domenico G, Scandola M, Zavattini G. YAP-PET: first results of a small animal positron emission tomograph based on YAP:Ce finger crystals. IEEE Trans Nucl Sci. 1998;45:3105-3108.
6. Jeavons AP, Chandler RA, Dettmar CAR. A 3D HIDAC-PET camera with submillimetre resolution for imaging small animals. IEEE Trans Nucl Sci. 1999; 46:468-473.

7. Correia JA, Burnham CA, Kaufman D, Fischman AJ. Development of a small animal PET imaging device with resolution approaching $1 \mathrm{~mm}$. IEEE Trans Nucl Sci. 1999;46:631-635.

8. Tai Y, Chatziioannou A, Yang Y, et al. MicroPET II: design, development and initial performance of an improved microPET scanner for small-animal imaging. Phys Med Biol. 2003;48:1519-1537.

9. Rouze NC, Schmand M, Siegel S, Hutchins GD. Design of a small animal PET imaging system with 1 microliter volume resolution. IEEE Trans Nucl Sci. 2004; 51:757-763.

10. Vaska P, Woody CL, Schlyer DJ, et al. RatCAP: miniaturized head-mounted PET for conscious rodent brain imaging. IEEE Trans Nucl Sci. 2004;51: 2718-2722.

11. Miyaoka RS, Janes ML, Lee K, Park B, Kinahan PE, Lewellen TK. Development of a Prototype Micro Crystal Element Scanner (MiCES): QuickPET II. Mol Imaging. 2005;4:117-127.

12. Phelps ME. PET: the merging of biology and imaging into molecular imaging. J Nucl Med. 2000;41:661-681.

13. Weissleder R, Mahmood U. Molecular imaging. Radiology. 2001;219:316-333.

14. Vaalburg W, Hendrikse NH, de Vries EF. Drug development, radiolabelled drugs and PET. Ann Med. 1999;31:432-437.

15. Larobina M, Brunetti A, Salvatore M. Small animal PET: a review of commercially available imaging systems. Curr Med Imaging Rev. 2006;2:187-192.

16. Tai Y-C, Laforest R. Instrumentation aspects of animal PET. Annu Rev Biomed Eng. 2005;7:255-285.

17. Stickel JR, Cherry SR. High resolution PET detector design: modeling components of intrinsic spatial resolution. Phys Med Biol. 2005;50:179-195.

18. Levin CS, Hoffman EJ. Calculation of positron range and its effect on the fundamental limit of positron emission tomography system spatial resolution. Phys Med Biol. 1999;44:781-799.

19. Tai Y-C, Wu H, Pal D, O'Sullivan JA. Virtual-pinhole PET. J Nucl Med. 2008;49:471-479.

20. Wu H, Pal D, O'Sullivan JA, Tai Y-C. A feasibility study of a prototype PET insert device to convert a general purpose animal PET scanner to higher resolution. J Nucl Med. 2008;49:79-87.

21. Tai Y-C, Ruangma A, Rowland D, et al. Performance evaluation of the microPET-Focus: a third generation microPET scanner dedicated to animal imaging. J Nucl Med. 2005;46:455-463.

22. Janecek $\mathrm{M}, \mathrm{Wu} \mathrm{H}$, Tai YC. A simulation study for the design of a prototype insert for whole-body PET scanners. IEEE Trans Nucl Sci. 2006;53:1143-1149.

23. Pal D, O'Sullivan JA, Wu H, Janecek M, Tai Y-C. 2D linear and iterative reconstruction algorithms for a PET-insert scanner. Phys Med Biol. 2007;52: 4293-4310.

24. Casey ME, Gadagkar H, Newport D. A component based method for normalization in volume PET. Proceedings of the Third International Meeting on Fully Three-Dimensional Image Reconstruction in Radiology and Nuclear Medicine. Aix-les-Bains, France: IEEE; 1995:67-71.

25. Daube-Witherspoon ME, Muehllehner G. Treatment of axial data in threedimensional PET. J Nucl Med. 1987;28:1717-1724. 